\title{
The peculiarity of a nervous pulse propagation
}

\author{
Mileta Arakelyan, Hakob Kanoyan \\ Yerevan State University, Dept. of Physics, Yerevan, Armenia
}

Email address:

marakelyan@ysu.am (M. Arakelyan), hakob91@gmail.com (H. Kanoyan)

To cite this article:

Mileta Arakelyan, Hakob Kanoyan. The Peculiarity of A Nervous Pulse Propagation, European Journal of Biophysics. Vol. 1, No. 1, 2013, pp. 1-5. doi: 10.11648/j.ejb.20130101.11

\begin{abstract}
In the work, transmission of nerve impulses along nerve fibers is simulated. Research is being conducted in the framework of the electrical theory of propagation of the action potential. The soliton approach is used. The numerical experiment on the evolution of the nerve impulse and the laws of motion is conducted. It is shown that the stable form of nerve impulses solitons is realized under different initial conditions. The threshold character of occurrence of nerve pulse is simulated. It is shown that the number of pulses produced changes depending on the degree of nonlinearity: with strong initial excitation of large amplitude soliton is unstable; it breaks up into a multitude number of solitons with small amplitudes. In this case, the greater the nonlinearity parameter, the greater the number of births of solitons. Unusual solitonlike regimes of interaction of nonlinear pulses excitations are illustrated; in certain anomalies, colliding nerve impulses are reflected instead of their usual quenching. The possibility of the decay of the nerve impulse at the bifurcation of the nerve fibers or the appearance of heterogeneity of the passing of a nerve impulse (the presence of dissipation in the environment) is investigated. Physical modeling allows obtaining and studying the analytical results and elucidating the physical principles of biological processes.
\end{abstract}

Keywords: Soliton, Nervous Pulse, Nonlinear, Korteweg De Vries Equation

\section{Introduction}

To coordinate work of the different systems in the human body requires harmonious work of its most complex system - the nervous system. Its basic function is the propagation of an electric pulse through the nerve fiber. Physiological studies have shown that propagation of a nervous pulse through neural nets has autowave character. $\mathrm{T}$. Heimburg and A.D. Jackson proposed a new neural theory called Soliton theory. In this theory, the nerve conduction is proposed as a density wave. [1] B.Lautrup, R.Appali and others [2] examined a class of solitons, obtained from a generalization of the Boussinesq equation. Soliton model is based on the propagation of a localized density wave in the axon membrane. This allows simulating such processes using Soliton Theory, which describes the form and velocity of propagation of the nervous pulse, stability of its characteristic parameters in time, and process locality with perfect precision. [3]

According to electrical theory, the process of transmission of the pulse along the nerve fiber is a process of propagating the action potential: the nerve fiber extends the electrochemical reaction, which generates a running impulse of voltage. The nerve pulse or action potential propa- gates through the nerve fiber without decay and with constant velocity. An impulse can be formed and spread because the nerve fiber contains a nonlinear element, which suppresses small deviations from normal state and strengthens big ones. In this case, the nonlinear dependence of the membrane permeability (through which the nerve impulse is spread) from the momentum and the diffusion of ions across the membrane are balanced. In the paper [4] is shown that nonlinearity can actually balance diffusion and, as a result, a running solitary wave with constant speed and shape can occur, i.e. a soliton. Thus a nerve impulse is an extension of an action potential. Physiological studies have shown that propagation of a nervous pulse through neural nets has autowave character. This also allows simulating such processes using Soliton Theory which describes the form and velocity of propagation of the nervous pulse, stability of its characteristic parameters in time, and process locality with very good precision. [5]

\section{Theory and Numerical Experiment}

Since the nerve impulse is resistant, it extends without distortions and significant loss of energy; its movements can be modeled as a Korteweg de Vries soliton. In this case it is described by the nonlinear differential equation [6]: 


$$
\frac{\partial u}{\partial t}+\beta \frac{\partial^{3} u}{\partial x^{3}}+u \frac{\partial u}{\partial x}=0
$$

where the second term characterizes a weak (cubic) dispersion, and the third term describes nonlinearity. The solution of this equation has the following form,

$$
u(t, x)=\frac{u_{\max }}{\operatorname{ch}^{2}\left(\frac{x-v t}{\Delta}\right)}
$$

where $u$ is the action potential, $u_{\max }$ is the amplitude of the action potential (in our case of the nerve pulse), $v$ is the velocity

$$
\left(v=u_{\max } / 3\right)
$$

, and $\Delta$ is determined from the ratio

$$
\Delta=\sqrt{12 \beta / u_{\max }}
$$

From (2a) it follows, that the propagation velocity of a pulse increases with increase in the amplitude, whereas from $(2 b)$ it follows that the soliton width decreases with the increase in the amplitude. Therefore, the solitons line up in the order of decreasing amplitudes and growing widths "Fig. 1".
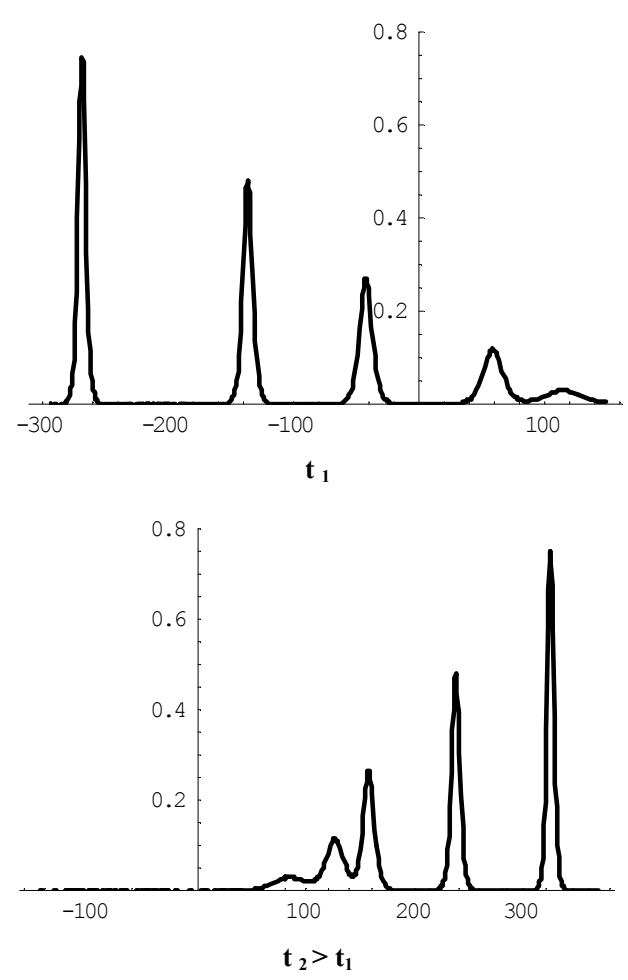

Figure 1. Soliton distributions at different times. (Numerical experiment).

We performed a numerical experiment of formation, evolution and movement mechanism of solitons. The stable form of solitons can be obtained with different initial conditions.

We chose the initial perturbation in two forms:

$$
u(x, 0)=u_{0} \exp \left(-\frac{x^{2}}{l^{2}}\right)
$$

(where $l$ is the linear size of initial perturbation) and a hyperbolic cosine. However, regardless of the character of initial perturbation, over time the pulses transform into a soliton. Obviously, the initial conditions are connected with the signal received from the brain through conducting ways and depend on the state of the organs or the mental state of the person. ( These data are consistent with [6] )

English physiologists A. Hodgkin and A. Huxley have developed a theory (based on numerous experiments) where they concluded that the shape and the speed of an impulse don't depend on the stimulus of the nerve. If the stimulus is high, several consecutive impulses are produced, while if it is low, no impulse will be produced through the nerve; the minimal power of stimulus to produce an impulse is called threshold. Each impulse transfers one unit of information and the receiving devices count the number of elementary particles of information received and the time it took [7]. All this reminds of the movement of solitons. In paper [8] Hodgkin-Huxley and Soliton theories are described and a theoretical comparison has been carried out throughout the analysis of the theories and models.

In the Korteweg de Vries equation the degree of nonlinearity is characterized by a dimensionless parameter $\sigma=l\left(u_{0} / \beta\right)^{1 / 2}$. The numerical experiment showed that at $\sigma \leq \sigma_{\text {min }}$ the solitons are absent,and instead sine wave oscillations arise "Fig. 2".

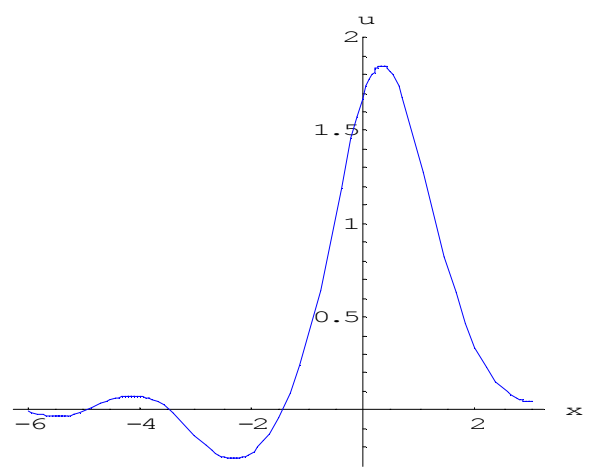

$\mathbf{a}$

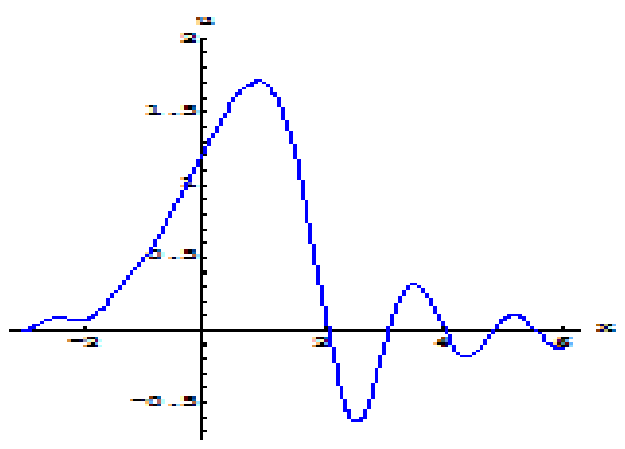

b

Figure 2. Sine wave oscillations. $\sigma \leq \sigma_{\min }$ 
From "Fig 2a" it is visible that solitons are absent, but to the left of an initial impulse there are oscillations. This case is realized at $\beta>0$. At $\beta<0$ ("Fig 2b") oscillations arise on the right. The received result follows from Korteweg de Vries equation.

We investigate character of the solution of the equation (1) depending on values $\sigma$. For $\sigma=5.9$ we studied evolution of an initial impulse for times $t_{1}<t_{2}<t_{3}$. Thus the initial impulse moves to the right, the first solitons amplitude grows over time, at $t_{2}$ the second soliton is born whose amplitude also grows over time; at $t_{3}$ we have 2 solitons, and the amplitude of the first one is higher than the amplitude of the second one. At $\sigma=9$ and the same initial condition the decision for the same values of time is received. It can be seen from the figure that 2 separate solitons on the right and the third, a new one, on the left are visible. Thus, with increase of $\sigma$ at an identical initial impulse the number of born solitons increases "Fig. 3".

It is known that in vivo (living organism) nerve impulses moving along the axon toward each other collide and annihilate each other, which provides one-way transmission of signals from receptors in the central nervous system. However, the works of Aslamanidi O.V., Mornev O.A. [9,10] are based on numerical experiments with Hodgkin-Huxley equations predict a new effect: for some values of the parameters colliding nerve impulses may reflect. It is shown that in the soliton mode, the propagating impulse is a "doublet" consisting of a high-amplitude impulse-leader and an accompanying low-amplitude wave. The first collision of the leaders causes annihilation, and the second collision of the low-amplitude waves leads to summation. As a result, at the point of collision the potential reaches the above-threshold value, causing regeneration of "doublets" that become scattered in different directions.
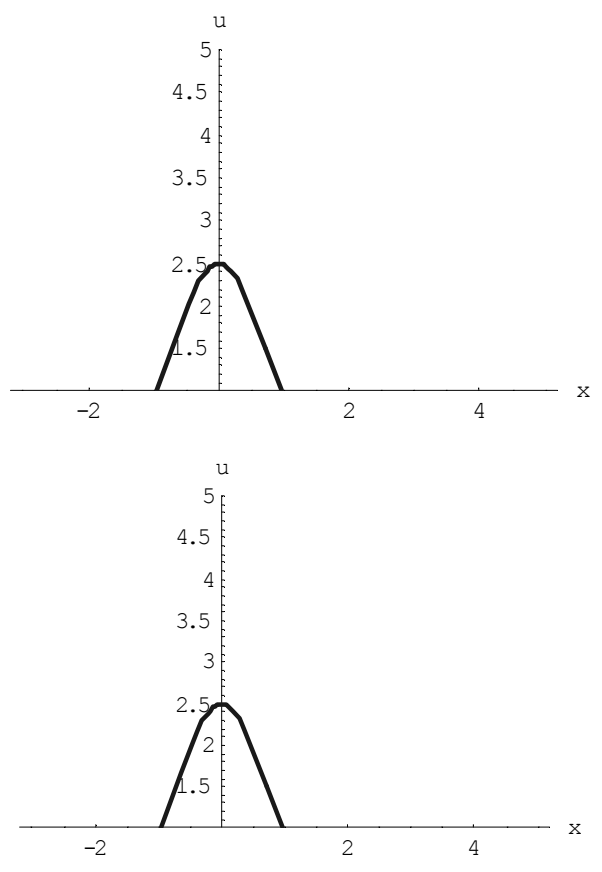

$\mathbf{t}_{1}$
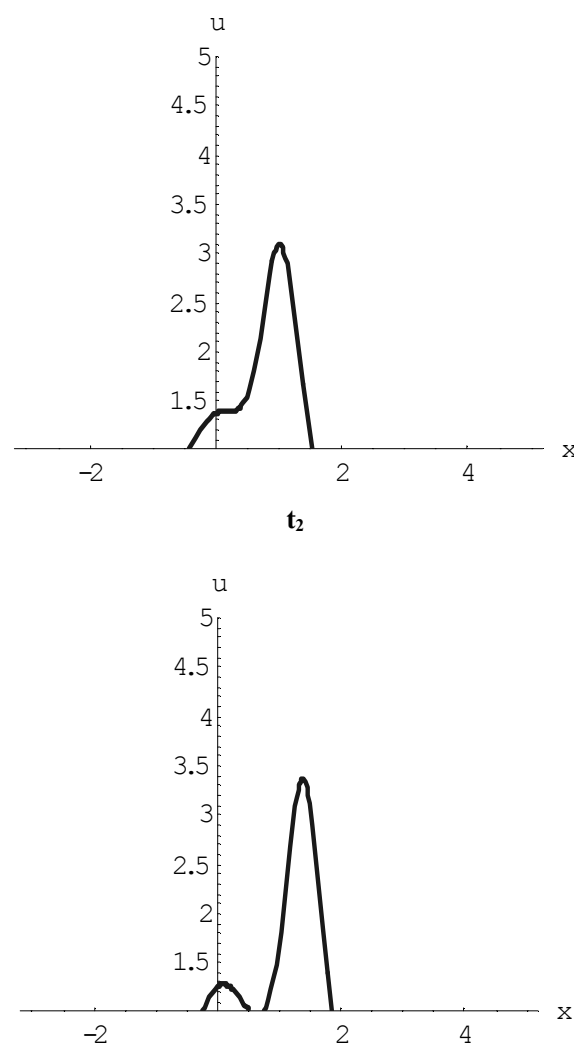

$t_{3}$

$\sigma=5.9$

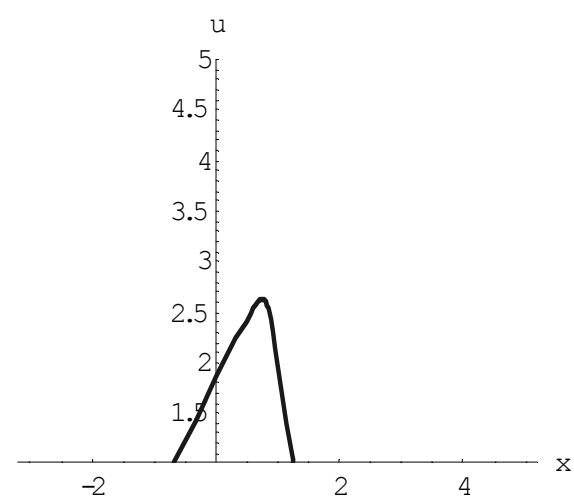

$\mathbf{t}_{1}$

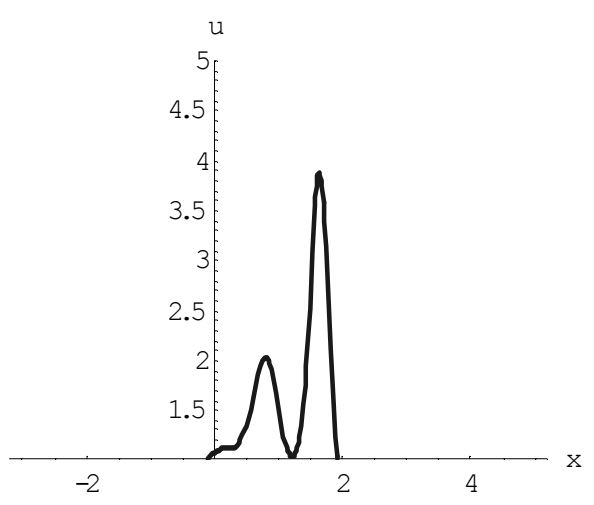

$\mathbf{t}_{2}$ 


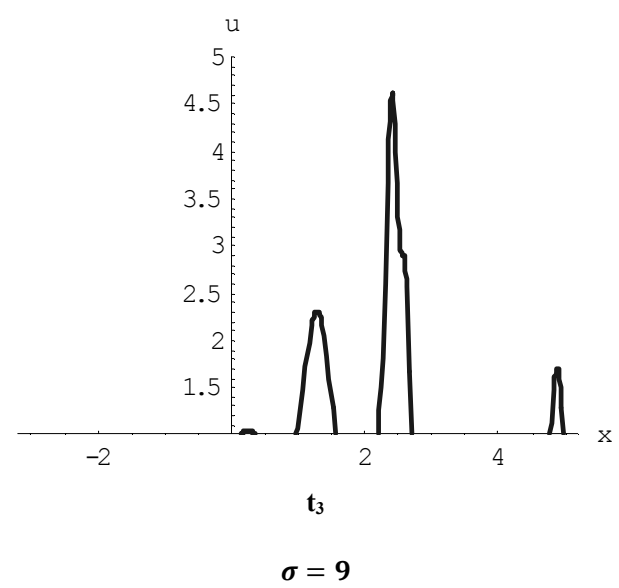

Figure 3. The different number of originating solitons for different values of nonlinear parameter.

We have modeled the collision of two solitons occurring in a similar mode, on the basis of Korteweg de Vries equation "Fig. 4".

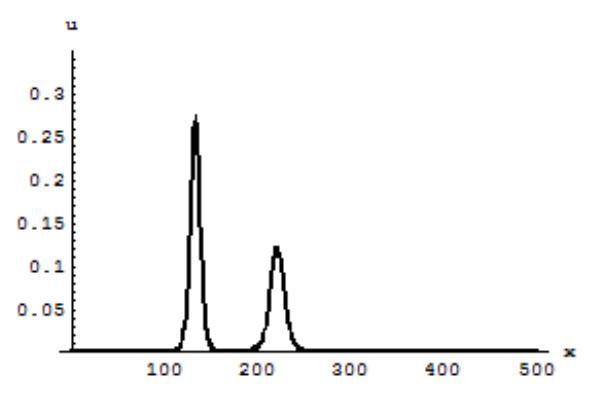

$\mathbf{t}_{1}$
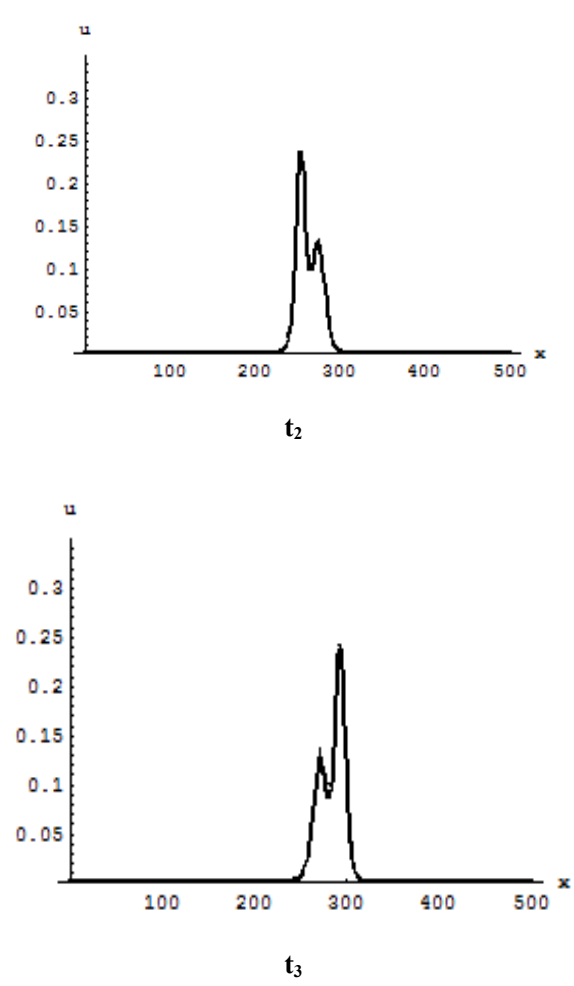

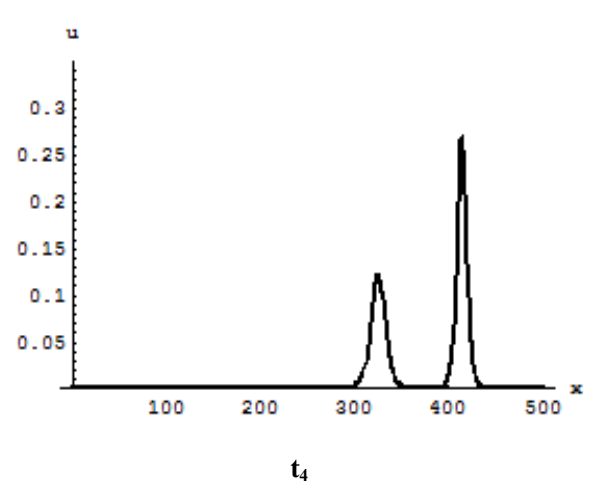

Figure 4. Collision of two solitons. The figures show successive moments of time.

The analysis of such regimes of interaction of nonlinear pulse can be useful in deciphering the mechanisms of cardiac arrhythmias. It is known that the Korteweg de Vries soliton gradually slows down under friction, reducing height and becoming blurred. The lifetime is inversely proportional to the force of friction. Analogically, when the cross section is changed or nerve fibers are bifurcated, the passage of nerve impulses may become difficult or even completely blocked. In the equation, this can be considered as the presence of friction. [6] We have also investigated the solitons' evolution in the presence of friction. When taking friction into account, the Korteweg de Vries equation becomes the form

$$
\frac{\partial u}{\partial t}+u \frac{\partial u}{\partial t}-u \frac{\partial^{2} u}{\partial t^{2}}+\beta \frac{\partial^{3} u}{\partial x^{3}}=0
$$

where $\mu$ is the coefficient of friction. Suppose that at $\mathrm{t}=0$ we have an initial perturbation in the form

$$
u(x, 0)=u_{0} \operatorname{ch}^{-2}(x / l)
$$

At $\mu=2$ the numerical experiment was made for $\mathrm{t}=\mathrm{t} 1<$ $\mathrm{t} 2<\mathrm{t} 3<\mathrm{t} 4<\mathrm{t} 5$. "Fig.5a". Next, the same experiment was performed for $\mu=10$ and for the same values of time. As expected, attenuation occurs faster with with the increasing coefficient of friction. "Fig.5b".

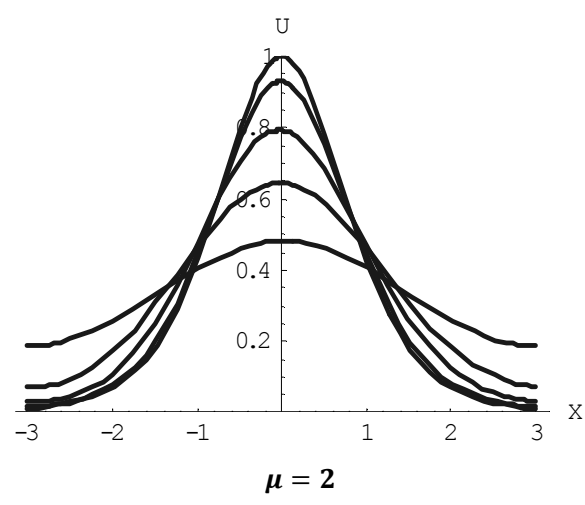

$\mathbf{a}$ 


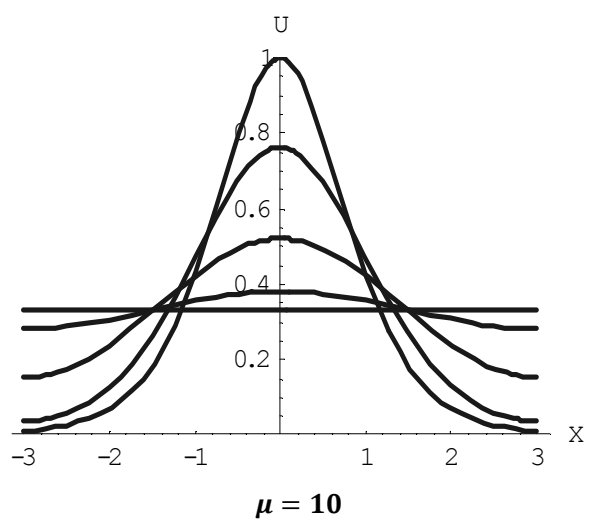

b

Figure 5. Solitons evolution at different coefficients of friction $\mu$ for the same values of time.

\section{Conclusion}

Thus, different regimes of propagation of nerve pulses can be realized under different conditions. Solitonlike solutions describe a wide range of phenomena that accompany the transmission of nerve pulses, heart rate, self-oscillation. The soliton models of processes make it possible to obtain adequate description and identification of the state of such systems as the conducting muscular and circulatory systems of the heart. [11] By changing parameters of the soliton model, it is possible to model the pathologies of nervous conducting ways of the vascular and muscular system of the heart. The soliton approach opens up large possibilities in the research into the complex processes in living organisms. Possible pathology in the vascular system changes spectral picture of the soliton waves and allows diagnosing a variety of diseases of nerve pathways and the circulatory system. Mathematical modeling provides the analytical results and the way to identify the physical principles of biological processes.

\section{References}

[1] T.Heimburg A.D.Jackson. On Soliton propagation in biomembranes and nerves. Proc.Nat1.Acad.Sci.USA, 102, 9790-9795, 2005.

[2] B.Lautrub, R. Appali, A.D.Jackson and T.Heimburg. The stability of Solitons in biomembranes and nerves.Eur.Phys.J E 34(6) (2011) 1-9.

[3] S.Rowlands. Is the Arterial Pulse a Soliton? J.Biol.Phys.v.10, p.199-200,1982.

[4] A.N. Kolmogorov, I.G.Petrowskij, N.C.Piskunov.Bull.of Moskow State University, 1,N6, 1937. R.A. Fisher. The wave of advance of advantageous genes// Annual Eugenics, 1937, v.7, p.255-369.

[5] Karl Lonngren, Alwyn Scott. Solitons in Action. Proceedings of a Workshop, 1977.

[6] Markin V S, Pastushenko V F, Chizmadzhev Yu A "Physics of the nerve impulse" Sov. Phys. Usp. 20 836-860 (1977)

[7] A.Hodgkin, A.Huxley, J.Physical.(LND), 117, 500, 1952.

[8] R.Appali, S. Petersen and van Rienen.A comparision of A.Hodgkin- A.Huxley and Soliton neural theorives. Adv.Radio Sci, 8,75-79, 2010.

[9] O.V.Aslanidi, O.A.Mornev, (1997), Can the colliding nerve pulses be reflected? Pis'ma Zh.Eksp.Teor.Fiz., 65,No.7, 553-558.

[10] O.V.Aslanidi, O.A.Mornev.Echo in excitable cardias Fibers.Mathematical Modelling Journal.v.11, No 9, p.3-22. 1999

[11] G.M.Aldonin. Soliton Models of Processes in Biostructures. Journal of Radio Electronics, N5, November 2006. 